\title{
DIVERSIDAD CULTURAL Y TECNOLOGÍA
}

\author{
Xabier Etxeberria ${ }^{1}$
}

Trabajo recepcionado: diciembre 2006

Trabajo aceptado: junio 2007

\begin{abstract}
RESUMEN
Este trabajo se plantea varias preguntas acerca de la diversidad cultural y la tecnología. ¿Qué lugar ocupa la tecnología en el fenómeno de la diversidad cultural y con qué dinámicas? Contra lo que pudiera parecer en un primer momento, ¿es un elemento decisivo a la hora de plantearse éticamente las relaciones entre los diversos grupos culturales? ¿Cómo habría que abordarlo cuando se pretende una gestión respetuosa y solidaria de las diferencias culturales? Son estas las tres preguntas que guían el presente artículo. Para afrontar las interrogaciones formuladas, este artículo expondrá los argumentos en dos grandes partes. En la primera, se tratará de explicitar qué lugar ocupa la tecnología en la producción y vivencia cultural, resaltando cómo ella está ineludiblemente abocada a la diversidad de sus expresiones. Una vez precisado el alcance de esta diversidad de la tecnología, en la segunda parte se analizará lo que ello supone y debe suponer para la relación entre culturas.
\end{abstract}

PALABRAS CLAVE: Diversidad cultural, tecnología, tecnociencia, filosofía de la ciencia.

\begin{abstract}
While producing an intercultural critique to modern science, the author proposes to reflect about science as a meditative thought that recovers its sense. Also, he looks for to where should be the point from which he is able to understand its meaning and role in view of science, taking consciousness about going beyond critique. Therefore, it is not enough to censor or show the deviations of applying science but to exceed the horizon and placing science in its place. However, to know which the place that science belongs is, implies recognizing the meaning of human knowledge and the cognitive activity within the general development of life. In other words, to know what we are supposed to have in our being, and how and when we are expected to be. In sum, this endeavor is about knowing our place and which sense would spring from that point, which is necessary to decide the place that science has in human life.
\end{abstract}

KEYWORDS: science-knowledge, intercultural philosophy, wisdom.

1 Universidad de Deusto (Bilbao) 
Revista CUHSO volumen $14 \mathrm{n} \div 1$

1. El lugar de la tecnología en la cultura y su apertura a la diversidad

Para aclarar adecuadamente el lugar de la tecnología en la cultura, es preciso describir el abanico de aspectos que esta implica, a fin de insertar a la primera dentro de ellos. Pues bien, en toda cultura se da una articulación compleja de tres niveles ${ }^{2}$, que vienen a ser el esquema formal compartido por todas, que luego se materializa de maneras diferentes en cada una de ellas, expresándose de este modo la diversidad cultural. Comencemos explicitando los niveles, para pasar luego a describir sus imbricaciones.

A) Ios niveles:

1. Tenemos, en primer lugar, el nivel de los instrumentos. Puede ser definido como el conjunto de medios y mediaciones para crear bienes y ofrecer servicios. Eso significa que valen en función de otro aspecto, que valen, en última instancia, en función de lo que es fin en sí. Desde luego no puede ignorarse que hay una tendencia a hacerlos fines, a subordinar nuestra vida a la posesión de los mismos.

Estos instrumentos implican saber empírico aplicado. Esto es algo que se expresa de modo sumo en la circularidad creativa que se está dando en el complejo tecnocientífico; gracias al saber científico hemos creado una imponente tecnología, pero a su vez, gracias a la tecnología de la que vamos disponiendo, podemos hacer nuevos hallazgos científicos.

La tecnología -el tema que propiamente nos ocupa- tiene su lugar decisivo en este nivel, aunque pueda proyectarse en los otros. La defino someramente apropiándome de una propuesta de quintanilla. El sistema tecnológico, dice este autor, es un sistema planificado de acciones (intencionales y racionalmente estructuradas) que involucran artefactos. En efecto, 1) se trata de un sistema -no de elementos sueltos sin relación- dinamizado por acciones intencionales, esto es, dirigidas al logro de un fin -no se trata de una mera colección de instrumentos-; 2) implicando el ejercicio de una racionalidad que debe ser definida como instrumental: aquella que se aplica al discernimiento de los medios en vistas al logro de fines sobre los que no se discierne; 3) involucrando artefactos, es decir, instrumentos hechos como tales por el ser humano.
Con la invención y el uso de la tecnología se persigue, en principio, satisfacer necesidades muy variadas (como alimentación, abrigo, salud, seguridad, información, movilidad...), Pero también deseos; e igualmente potenciar las capacidades de los usuarios. En esta somera relación de finalidades se intuyen ya las grandes ambigüedades de la tecnología.

- En primer lugar, no es lo mismo perseguir con ella la realización de las necesidades ligadas a la condición humana, como tales limitadas y satisfacibles, que perseguir colmar los deseos, como tales insaciables. Acabo de indicar que la razón tecnológica no discierne sobre los fines, no discierne respecto a la definición de necesidades y deseos; por eso, precisamente, toca a la racionalidad ética y de sentido, la que se aplica a los fines, aclarar lo más posible cuándo hay necesidades que deben satisfacerse con la tecnología y cuándo hay deseos que, más bien, deben ser moderados en espíritu de sobriedad: los excesos tecnológicos, con su impacto negativo, tienen en buena parte su origen en su alineamiento con la satisfacción de deseos humanos estimulados sin medida. Hay que ser conscientes de que no es fácil hacer la delimitación precisa entre deseos y necesidades, que depende, además, en cierta medida, de las culturas y sus momentos históricos ${ }^{3}$, pero es muy importante distinguirlos en lo fundamental.

- En segundo lugar, también aparece una relevante ambigüedad en el hecho de que la tecnología potencia las capacidades de quienes la usan. Porque puede potenciar tanto nuestras capacidades de construcción individual y colectiva -capacidad de las personas- como las de destrucción -capacidad frente a las personas, contra ellas-. Hay instrumentos tecnológicos especializados en una u otra forma de capacitación (tecnología de elaboración del pan / pistolas), pero con frecuencia la ambigüedad que resalto anida en el propio instrumento, utilizable con fines varios. A partir de esta constatación, la pregunta por el tipo de tecnologías que estamos impulsando, y por su uso en relación con la convivencia y la construcción de lo humano, emerge con fuerza.

- Estas dos ambigüedades, que acabo de presentar, ponen de manifiesto la importancia que, para modular la creación y uso de la tecnología, tienen los ideales

\footnotetext{
2 Me inspiro en una propuesta de Paul Ricoeur, pero haciendo amplios desarrollos propios.

3 Por poner un ejemplo evidente: es indudable que en nuestra cultura saber leer y escribir es una necesidad perentoria, mientras que en las culturas ágrafas ni siquiera cabe plantearla.
} 
culturales de realización de las personas. Hay culturas que hacen propuestas más centradas en la satisfacción de las necesidades y la convivencia, mientras que otras estimulan la realización de los deseos y/ o la expansión dominadora. Como puede verse, la conexión entre la tecnología y la ética va configurándose, tanto en lo que esta tiene que ver con los horizontes de plenitud como en lo que tiene que ver con los ideales de convivencia.

Hay una tercera ambigüedad de la tecnología, que emerge a partir de su relación con el medio ambiente. Este es tanto una fuente de recursos cuya explotación facilita la tecnología -capacitación frente a la naturaleza y con frecuencia contra ella-, como un depósito paralosresiduos-con mucha frecuencia dañinosque la tecnología acrecienta. La ambigüedad está aquí en que el sistema tecnológico puede facilitar nuestra relación positiva con la naturaleza, no solo considerada como fuente de recursos necesarios para la satisfacción de nuestras necesidades, sino también como ámbito de contemplación (piénsese a qué podemos acceder gracias a un telescopio 0 a un microscopio); pero, igualmente, puede dinamizar nuestras tendencias dominadoras y destructivas respecto a ella. De nuevo, vuelve a aparecernos la importante conexión, que remite una vez más a la ética y al sentido de la realidad, entre sistema tecnológico y propuesta cultural global -relativa a los finesen la que el primero se sitúa: no va a ser lo mismo una propuesta que considere al ser humano en la naturaleza, impulsado por el ideal de armonizarse todo lo posible con ella, que otra que nos hable del ser humano frente a ella, con espíritu de dominio estricto.

2. Desarrollado con algo más de precisión el nivel de los instrumentos, por situarse en él nuestro tema, paso a presentar de modo más elemental los otros dos niveles existentes en toda cultura, comenzando por el de las instituciones.

Cabe entender, por instituciones, el conj unto de formas estructuradas de existencia social con las que se regulan normativamente las relaciones. De ellas destaco en concreto:

- Que están ligadas, para lo bueno y para lo malo, a las dinámicas de poder. Para lo bueno, en la medida en que acrecientan las posibilidades de intervención mucho más allá de lo que pueden hacerlo los individuos en cuanto tales. Para lo malo, porque las concentraciones de poder que suponen (dependiendo, por supuesto, de los tipos y modos de institución) pueden utilizarse para oprimir y marginar a los que ocupan en ellas lugares de subordinación.

- Que son necesarias, incluso ineludibles, para hacer viables y durables las propuestas diversas de realización colectiva. Precisamente por el poder opresor que puede anidar en ellas, cabe la ilusión de plantearse su desaparición, pero en realidad, a lo que podemos aspirar es a su transformación, precisamente para que cumplan bien su objetivo de facilitar y potenciar el vivir juntos.

Las instituciones posibles -y las que realmente se han dado y se dan- son de múltiples tipos: según los ámbitos de convivencia colectiva que regulen -familiares, religiosas, económicas, políticas, jurídicas, etc.-; Según las imbricaciones que se planteen entre ellas; y según la concreción que les den las diferentes culturas en sus diversos momentos históricos.

3. El tercero de los niveles presente en toda cultura es el ético-simbólico. Está constituido por el conjunto articulado de propuestas de sentido sobre la realidad (cosmovisión) y de orientación para la conducta (éthos). Hay que distinguir en él, a su vez, varios subniveles:

- En la superficie están las costumbres, que tienden a la inercia, esto es, que pueden continuar practicándose cuando se ha perdido el referente de sentido que las ideó y les dio vida (por ejemplo, es lo que pasa, al menos en muchas familias europeas, con diversos aspectos de la celebración de la navidad).

- Bajo el subnivel de las costumbres está el de las tradiciones o memoria viva de la comunidad. Hay que hablar de tradiciones en plural, con frecuencia tensionadas entre ellas (lo que, bien llevado, es ocasión de creatividad y riqueza), aunque esté permanente la tentación de reducirlas a una, que actúa entonces impositivamente.

- Como núcleo decisivo, tenemos el conjunto de símbolos y relatos que expresan el sentido de la realidad, los valores, las normas, los ideales de realización (también aquí en versiones plurales, aunque siempre alguna se plantee como dominante).

Históricamente, las propuestas religiosas han ocupado un lugar central, prácticamente en todas las culturas, en 
este nivel ético-simbólico. Con todas sus variedades interreligiosas y sus tensiones intrarreligiosas. Y con sus múltiples formas de insertarse en las culturas en su conjunto. De todos modos, no habría que reducir este nivel a ellas. $Y$ debe contemplarse la posibilidad de expresiones ateas o agnósticas del mismo (está en discusión si es a este agnosticismo a lo que dominantemente se va avanzando en las sociedades europeas).

4. Para tener una visión más completa de las dimensiones estructurales de las culturas, conviene hacer un par de observaciones a la presentación que se ha hecho de los niveles.

- La primera de ellas es que debe contemplarse en toda su importancia un elemento transversal a los mismos que está resultando decisivo para la configuración de las identidades culturales: la lengua o idioma en el que un grupo habla y que considera propio.

- La segunda observación tiene que ver con el hecho de que la presentación en niveles supone una descripción formal de las culturas, que es estática o sincrónica. Dado que toda realidad, también la cultural, es dinámica, hay que insistir en que en la concreción de los niveles se dan constantes transformaciones, debidas tanto al dinamismo de la interacción entre ellos, que resaltaré a continuación, como del dinamismo histórico, del que quiero dejar ahora constancia. Lo que supone: 1) que las culturas son realidades históricas sujetas a evoluciones complejas, con estímulos tanto intraculturales como interculturales; 2) que los grupos culturales tienden a considerarse sujetos históricos, siendo su identificación con una determinada historia -la suya- un elemento decisivo de configuración de su identidad.

B) Ia articulación compleja de niveles:

1. Ya he adelantado que los niveles descritos de las culturas no son compartimentos estancos, funcionan articuladamente, con imbricaciones complejas. Estas pueden darse en múltiples direcciones:

- La lógica -la ética- pide que el nivel ético-simbólico, situado en sí en el reino de los fines, regule crítica y creativamente las instituciones, así como los estímulos, procesos y usos de la producción instrumental, que en definitiva constituyen el reino de los medios.

- Pero a su vez, no puede ignorarse la posibilidad de que las instituciones, autonomizadas y centradas en el poder, ejerzan un significativo control de la expresión del nivel ético-simbólico y de la producción tecnológica, poniéndolos a su servicio.

- E igualmente, el nivel tecnológico, cuando se hace sistema complejo, puede adquirir protagonismos, en su relación con los otros niveles, que no son los suyos propios. Pero dado que esto tiene que ver específicamente con nuestro tema, paso a desarrollarlo más extensamente.

2. ¿Cuáles son, pues, los procesos de imbricación de niveles en los que media la tecnología? Apuntan en varias direcciones.

En primer lugar, aunque la tecnología es en sí instrumental como ya he reiterado, en la medida en que está impulsada no solo por la abstracta satisfacción de necesidades y deseos, sino por un determinado mundo simbólico-ético (o al menos enmarcada en él) que las y los modula, acaba quedando contagiada por él de múltiples formas. Esto quiere decir que cada grupo cultural, con su núcleo ético simbólico específico, empuja también modos específicos hacia la producción tecnológica y los impregna de formas diferentes. Por ejemplo, para incitar la invención y uso de la tecnología:

- No es lo mismo situarse dentro de una visión cultural de la realidad que considera a los humanos frente al mundo, que situarse en otra que los considera parte de él (tecnología que impulsa la explotación de la naturaleza frente a tecnología favorecedora de la convivencia armónica);

- No es lo mismo encontrarse dentro de una concepción cultural de la relación con el otro orientada a la hospitalidad, que encontrarse en otra en la que se propugna la expansividad de conquista (en la segunda, la tecnología armamentista será decisiva);

- No es lo mismo tener como referencia de sentido la autorrealización por la acumulación, que tenerla por la sobriedad (tecnologías encaminadas a la satisfacción de los deseos frente a tecnologías encaminadas a la satisfacción de las necesidades).

Las tres disyuntivas que presento no son hipotéticas, ya que cada cultura concreta se remite prioritariamente a una u otra de ellas, incidiendo, por tanto, en formas específicas de estimulación de la producción y utilización de la tecnología. 
Ensegundolugar, yen dirección inversa, cabe también la posibilidad de que se dé una cierta autonomización del nivel tecnológico al que luego se acomodan los otros niveles (sutil inversión de la relación medios-fines). Es lo que tiende a suceder cuando aparecen expresiones potentes de la tecnología (como el actual complejo tecnocientífico) o expresiones que por diversas circunstancias ejercen una fuerte fascinación (como ha sido en general el caso de la tecnología del armamento, o como los son actualmente ciertas tecnologías de la información). Advirtiendo de antemano, de modo genérico, que esta tendencia está modulada diferentemente según las culturas y según su momento histórico, pongo algunos ejemplos de las influencias indebidas de lo tecnológico potente/fascinante en los otros niveles, que están siendo muy reales:

- Estructurar la masculinidad a imagen de lo que sugieren las tecnologías de la guerra, con la correspondiente orientación a la competitividad, la dominación y la violencia;

- Organizar la vida autorrealizada de acuerdo a la oferta tecnológica, como vida definida por la posesión del mayor número posible de artefactos actuales;

- Sufrir una crisis en el nivel de sentido de la realidad, por ejemplo, cerrándose a las realidades transempíricas, a lo divino, no a causa de determinados procesos de interioridad, cuanto por el prestigio que la tecnología da a lo empírico-utilitario, como tal ciego para lo trascendente;

- Caer en la ilusión de que se puede y se debe realizar tecnológicamente todo lo que socialmente es posible, apoyándose en el supuesto ilusorio de que lo tecnológico por sí mismo es liberador.

Una tercera imbricación de lo tecnológico en los otros niveles, que conviene poner de manifiesto, es la siguiente: ciertos modos de complejización de las instituciones (políticas y económicas en especial, pero también de otras como las religiosas), así como de fortalecimiento y uso de su poder, están ligados a la incorporación a las mismas de determinadas tecnologías. Aquí es la institución - o algunos sectores de la misma- la que se fortalece por la tecnología que incorpora. Piénsese, por ejemplo, en las modernas tecnologías de la información y la comunicación (tic). Aunque son tan decisivas, que se están generando influencias en las dos direcciones: las instituciones acrecientan sus posibilidades gracias a ellas (algunas, como los medios de comunicación, identificándose prácticamente con ellas), pero a su vez, de algún modo, se acomodan internamente a lo que ellas exigen. Esto nos muestra que cabe una circularidad de potenciación mutua instituciones/ tecnologías, que las diversas culturas tienden a plasmar de formas diferentes, según, por ejemplo, la concepción del poder implicada en su núcleo ético-simbólico.

Un cuarto aspecto, que también es significativo, tiene que ver con la juridificación -nivel institucional- de la propiedad de los hallazgos tecnológicos, con lo que supone de cara a la estimulación, la invención, la difusión y la utilización de los mismos. Aquí vuelven a aparecer variaciones según las culturas y sus momentos históricos: en unos casos se postula decididamente la propiedad colectiva anónima, en otros la propiedad individual, en otros la propiedad institucional, etc. Habría que decir al respecto que es legítima la pluralidad, con tal de que cuando interactúan enfoques diversos no se generen opresiones, y con tal de que las formas de propiedad que se estimulen se muestren compatibles con el destino universal de los bienes, como decía tomás de aquino. Pero estas consideraciones nos introducen ya en el segundo apartado que anuncié al comenzar, el de las relaciones entre culturas mediadas por la tecnología.

\section{La diversidad cultural tecnológica y la relación entre culturas}

1. Conviene que abordemos este tema comenzando por precisar las modalidades de relaciones entre culturas y cómo puede mediar en ellas la tecnología.

En principio, las relaciones entre grupos culturales pueden ser de tres tipos:

- De dominio, en sus dos variantes de explotación y de marginación.

- De armonización, que en su nivel más básico supone el respeto y en su nivel superior la interrelación positiva para las partes.

- De ambigüedad: es el caso de la asimilación de una cultura por otra, que normalmente es una forma de dominación, pero, al menos en teoría, si fuera asimilación libremente consentida, sería algo aceptable.

Pues bien, todas estas formas de relación están mediadas por la tecnología. Basta con citar algunas de las modalidades más destacadas de esta -reconociendo de antemano 
que las fronteras entre ellas no son nítidas-, para que su función mediadora con una u otra forma de relación entre grupos culturales se muestre evidente:

- Tecnologías de la guerra.

- Tecnologías de la movilidad.

- Tecnologías de la información y la comunicación.

- Tecnologías para la producción de bienes, para la satisfacción de las necesidades básicas (alimentación, salud....).

La orientación positiva, negativa 0 ambigua de las mismas, en los procesos de relación entre culturas, depende en parte de la propia naturaleza de la tecnología, pero aún más decididamente de la utilización que se pretende hacer de ella, de la intencionalidad con que se las carga. De ahí la necesidad de analizar éticamente las tecnologías y sus usos, cuando se proyectan en las relaciones intergrupales.

2. Un aspecto concreto en la relación entre culturas, que afecta directamente a la tecnología, tiene que ver con el respeto al derecho a la propiedad tecnológica. Hoy en día, con la importancia que han adquirido, en los marcos de la globalización, los derechos de propiedad intelectual, se nos muestra como una cuestión ética básica. Téngase presente, en efecto, que estos derechos son precisamente los que ofrecen seguridad jurídica a los titulares de patentes y marcas.

Luego, hay que comenzar constatando que en la actualidad la regulación de este derecho sigue teniendo las ambigüedades propias de los regímenes de propiedad. En principio, el ejercicio y cumplimiento del mismo depende de la regulación jurídica de la propiedad que se tenga y de la capacidad para hacerse efectiva que esta muestre, tanto a nivel intraestatal como a nivel interestatal. Siendo esto así, y existiendo a su vez pluralidad en las concepciones de la propiedad (individual/colectiva, privada/pública), Io lógico sería que los grupos humanos implicados por esta diversidad de concepciones, eco de la diversidad de culturas, participaran equitativamente en la especificación de esa regulación jurídica:

- Teniendo cada uno de ellos espacio propios autorregulados de acuerdo con sus concepciones de propiedad (ámbito intracultural);
- Y estableciendo a su vez procesos dialógicos equitativos para llegar a acuerdos en aquellos ámbitos y modos de regulación de la propiedad que puedan afectar conjuntamente a colectivos con concepciones diversas de la misma (ámbito intercultural: en unos casos dentro de un Estado, en otros en la comunidad internacional en su conjunto).

Como sabemos muy bien, no es precisamente esta dinámica la que prevalece. Dentro de los Estados y en la comunidad internacional, la expresión jurídica de la propiedad, aplicada a la invención o descubrimientotecnológico, estádecisivamente marcada por la visión de la cultura occidental, que privilegia la propiedad individual, con determinados controles públicos y con algunos espacios para la propiedad pública, variables según los países. Esto significa que los colectivos culturales que no participan de esta visión están en situación de desventaja injusta, a la que se le añade con frecuencia la de dominación bajo amparo jurídico.

3. Esta desventaja y dominio se ejemplifican muy bien con el caso de los conocimientos tradicionales de los pueblos indígenas, conocimientos especialmente asociados a la biodiversidad. Respecto a esta cuestión, en efecto, se pueden hacer las siguientes consideraciones:

Hay que comenzar resaltando que estos pueblos viven en unos territorios que concentran el grueso de la biodiversidad existente en nuestro planeta, y que tienen respecto a ella una significativa sabiduría con proyección tecnológica. Hasta hace poco, esta biodiversidad y conocimiento no habían interesado prácticamente a la economía y la industria que manejan el grueso de la tecnología, pero en estos momentos son una referencia cada vez más importante para nuevos procesos en los que esta tecnología no incide ya preferentemente en la materia inerte, cuanto en la materia viva (biotecnología, industria farmacéutica, etc.).

Ante este dato, hay que constatar que las regulaciones intraestatales e interestatales de los derechos de propiedad intelectual, e incluso algunos convenios internacionales sobre la biodiversidad hacia los que se está avanzando, siguen exponiendo al patrimonio tecnocientífico de los pueblos indígenas a las siguientes prácticas violentadoras: 
- Respecto a sus concepciones de la propiedad aplicadas en este caso al saber tecnocientífico: las de estos pueblos son en general intergeneracionales -anónimas- y con una clara perspectiva colectiva, mientras que lo que internacionalmente se privilegia aún es la perspectiva privada con modulaciones y espacios públicos.

- Respecto a los regímenes de la propiedad intelectual de patentes, marcas y derechos de obtentor (en variedadesvegetales): hay una fragmentación de referentes jurídicos, que también les resulta perjudicial, por las dificultades que tienen para moverse con autonomía soberana dentro de ellos.

- Por último, siguen dándose expresas usurpaciones de hallazgos tradicionales de los pueblos indígenas, relativos, por ej emplo, a la biomedicina. Se ha desarrollado ya una cierta conciencia mundial al respecto, pero no es aún suficiente para erradicar estas prácticas, para dar pleno y efectivo amparo jurídico a los derechos indígenas de propiedad sobre sus saberes tradicionales.

No debe extrañarnos, por tanto, que estos pueblos, en la misma medida en que van tomando conciencia de su identidad y de los derechos ligados a ella, reivindiquen en concreto derechos colectivos de propiedad sobre su patrimonio intelectual, a fin de poder proteger y disfrutar de sus conocimientos y sus recursos biológicos, partiendo de la afirmación de su soberanía respecto a ellos. Precisamente por la ligazón de estos saberes con el territorio que habitan (con su biodiversidad) y con la historia colectiva (con el saber intergeneracionalmente generado y transmitido), tienden con frecuencia a reivindicar estos derechos intelectuales colectivos como prolongación de sus derechos territoriales e históricos, o si se quiere, enmarcados en estos. En principio, es de justicia apoyar esta pretensión como situada dentro de la legitimidad que dan los derechos humanos.

Si se acepta esta conclusión, como entiendo habría que hacer, debería traer como consecuencia el reconocimiento y respeto, por parte del sistema oficial de propiedad intelectual, del derecho consuetudinario que regula la titularidad colectiva. Lo que debería significar la creación de un sistema específico de protección de la propiedad intelectual indígena.
Por último, es cierto que habría que compaginar este modo de propiedad, como cualquier otro, con el bien común de la humanidad. Que también aquí habría que aplicar la consideración antes hecha, al hilo de un pensamiento de Tomás de Aquino, de que las formas de propiedad que se estimulen tendrán que ser compatibles con el destino universal de los bienes. No creo que los pueblos indígenas tengan especial problema en asumir la búsqueda de acuerdos en vistas a ello, si ven a su vez a lo que llamamos los pueblos de cultura occidental interesados en reformular de modo efectivo su derecho jurídico a la propiedad de modo tal que también se enmarque en el respeto al bien común (Téngase presente, para ver lo que esto podría significar, cómo el actual derecho de propiedad aplicado al campo farmacéutico, sigue frenando de modo inmoral -homicida- la difusión de medicamentos que podrían salvar miles y miles de vidas).

4. Tras estas consideraciones en torno al tema específico de la propiedad intelectual, voy a cerrar este artículo volviendo a un enfoque más general del tema de la relación entre grupos culturales y la tecnología, para ofrecer algunas observaciones que surgen cuando se tienen presentes las posibilidades de difusión y transferencia entre culturas de lo que son.

Hay que partir, para empezar, de la constatación de que la relación entre el nivel ético-simbólico (especialmente, elementos relevantes del núcleo del mismo) y el nivel institucional es compleja y no fácilmente transferible en el respeto. Aparece aquí toda una problemática en torno a la inculturación, especialmente importante en cuestiones como las religiosas, en la que no voy a entrar, porque desbordan los límites de este trabajo.

En cambio, y en principio, el nivel que puede transferirse con más facilidad entre culturas, en la aceptación consentida, e incluso entrar en una lógica de la acumulación interculturalmente compartida, es el instrumental-tecnológico. Ahora bien, para que este proceso no suponga dominación cultural hay que tener adecuadamente presente una serie de cuestiones que concreto del siguiente modo.

Cuando una cultura asume tecnología de otra, tiene alta probabilidad de asumir a su vez el contagio cosmovisional -positivo o negativo- que esta tiene. Cabe protegerse, frente a las dimensiones amenazadoras, con una adecuada inculturación, que pide a 
su vez, como condición de posibilidad, una significativa autonomía por parte del colectivo receptor. Y en cualquier caso, se precisa en sí un discernimiento, para saber qué se introduce y qué consecuencias puede tener.

Este discernimiento tendría que contemplar la posibilidad de frenar determinadas asunciones tecnológicas, o condicionarlas en sus formas a determinados requisitos. Ahora bien, hay que reconocer que hoy esta tarea es especialmente difícil debido al expansionismo, que parece imparable, de las tecnologías modernas. No puede ignorarse, en efecto, que el actual avance tecnológico, como posibilidad de poder y de producción, ejerce una poderosa fascinación (ambigua, si no es discernida críticamente) que le ayuda a difundirse en la asunción inconsciente de que es más fin que medio.

Estas consideraciones se aplican de modo especial a la tecnología de la información actual (occidental en su espíritu inicial). No se ofrece solo como tecnología, pues abre ineludiblemente la puerta a la información y la relacionalidad que posibilita, con las consecuencias de impacto cultural -positivo y negativo- que ello tiene. Precisamente por ello, hay que preguntarse qué regulaciones de la misma habría que hacer, pensando en el conj unto y, específicamente, en las culturas más propiamente receptoras. Tema ciertamente delicado, no solo por las dificultades objetivas que tiene, sino porque hay que decidir bien desde qué valores de referencia se hace esa regulación (no pueden ser, por ejemplo, los de posibilitar el mantenimiento del poder a costa de negar la autonomía individual y colectiva tal como está regulada en los derechos humanos).

Continuando con las tecnologías complejas, aunque más en general, hay que subrayar que se puede transmitir el artefacto o instrumento de un grupo cultural a otro, pero sin que se transmita el saber que supone ni su capacidad de producirlo. Esto supone, evidentemente, un claro riesgo, cuyo alcance debe ser medido con sensibilidad ética, de que la tecnología así transferida genere dependencia y dominación.

Un último bloque de comparaciones tiene que ver con la comparabilidad que puede realizarse entre grupos culturales, apoyada en su creatividad tecnológica.
- Dado que la eficacia de la tecnología respecto a lo que persigue es medible y comparable con facilidad en sus variaciones, tendemos a juzgar a las culturas en función de su potencial tecnológico bruto. Con esta perspectiva, la cultura occidental tiende a resultar ganadora. Ahora bien, si la referencia central deben ser los fines y no los medios, si la referencia debe ser la plenitud de realización y de sentido, ¿no sería más adecuado hacer apreciaciones no tanto (o al menos no primariamente) en función del avance en los medios -nivel instrumental-, cuanto en función de la definición y realización de los fines -nivel ético y simbólico-? La mayoría de los antropólogos defienden, creo que con razón, que hay que postular una inconmensurabilidad entre culturas (no una incomunicabilidad), Io que significa que no podemos establecer una especie de jerarquía valorativa global entre ellas, porque pediría que alguien externo a todas ellas, dotado de referentes aculturales incuestionables, la realizara, y no existe ni ese alguien ni esos referentes. Ahora bien, sí cabe hacer valoraciones sobre cuestiones concretas, con el criterio transcultural básico compartible de la dignidad del ser humano, y es aquí donde tenemos que elegir la perspectiva adecuada a la hora de hacer determinadas comparaciones.

- Por otro lado, incluso cuando se establece la comparación directamente entre tecnologías, debería valorárselas no tanto por su capacidad manipulativa en sí misma, cuanto por su adecuación para el logro de fines valiosos. Piénsese a este respecto en las tecnologías de impacto directo en la Naturaleza y sus consecuencias de cara a la conservación de los equilibrios ecológicos: una pala mecánica, ciertamente, moviliza muchísima más tierra en muchísimo menos tiempo que una pala manual, pero en ciertos ambientes y ciertos contextos, esta es preferible a la primera, que destruye todo ser viviente. La idea de fondo que quiero sostener, no es la de la vuelta romántica a un cierto primitivismo de la tecnología, sino la del discernimiento en torno al uso de la misma en función de sus ámbitos de aplicación. En ese momento, tecnologías oficialmente más modestas -antiguas y recientes-, volverán a ser apreciadas en lo que realmente valen.

Planteamientos como estos, por supuesto matizados en lo que tengan que ser matizados, ayudarán ciertamente a una respetuosa y empática relación entre grupos culturales cuando está mediada expresamente por la tecnología. A este obj etivo está orientado este trabajo. 